\title{
A Novel Frame Work for Detection of Chemically Ripened Mango Fruits Using Dominant Colour Descriptors
}

\author{
Laxmi $\mathrm{V}^{\mathrm{a}, 1}$ and Roopalakshmi $\mathrm{R}^{\mathrm{b}}$. \\ ${ }^{a}$ Dept of Information Science and Engineering BNMIT, Bengaluru, India. \\ ${ }^{\mathrm{b}}$ Dept of Information Science and Engineering AIET, Mangalore, India.
}

\begin{abstract}
Nowadays computer vision systems are widely used for identification, classification and grading of different kind of fruits. Existing research concentrates on features like size of the fruit, colour, shape and texture for classification and maturity detection of mango fruits.Colour of the fruit is one of the prominent feature, though a lot of effort is focused towardsidentification, maturity and defect detection of mango using colour features,less attemptsare made in the direction of the identification of artificially ripening of mango fruits.From another perspective very few attempts are concentrated towardsdeeper analysis of colour features of the fruits. In order to solve these issues this research paper proposes a new framework for detection of artificial ripening of mango fruit based on MPEG-7 colour descriptors. The proposed scheme includes three stages: first, a pre-processing stage consisting of masking, filtering, segmenting and cropping of an image followed by dominant colour extraction using dominant colour descriptors which are finally mapped with the help of clustering to identify the artificial ripening of mango fruit. The results of experimentsis carried out on two different datasets involving four types of mangoes which demonstrates robustness and efficiency of the proposed method against various other methods.
\end{abstract}

Keywords: Dominant colours,artificial ripening, computer vision,image processing.

\section{Introduction}

Mango (Mangiferaindica) is the national fruit of India and is deemed the king offruits on account of its nutritive value, taste, aroma and health promoting qualities. According to National Horticulture Board 2017, in India mango is grown in the prime area i.e. 2263 thousand hectares area where the production is around 19.687 million tons, thus providing about $8.7 \mathrm{MT} /$ hafruit production in the country. Mango is one of the major climatic fruit which thrives in a tropical climate and is loved for its lusciousness, exotic flavor and delectable taste throughout the world. It highly contains Vitamin C (15.1 mg/100g), minerals, lipids $(0.1 \mathrm{~g} / 100 \mathrm{~g})$ andproteins $(0.3$ $\mathrm{g} / 100 \mathrm{~g})$. The total sugars in mango reportedly consists of the total sucrose of different mango cultivars ranging between $10.00-17.30$ per cent[1,2].Mango is mainly seasonal fruit generally harvested in the month of April when weather is hot and humid which is generally picked at unripened stage and allowed to ripe naturally Maturing is a biological process which converts the fruit to bepalatable, edible and beneficial in the absence of disturbance for a considerable period of time [3]. Mango.

\footnotetext{
${ }^{1}$ Laxmi V, Dept of Information Science and Engineering BNMIT, Bengaluru, India.

E-mail: vlaxmiachar@gmail.com
} 
is a highly nutritious fruit, it is ripened by using some artificial ripening agents in most of the countries. Though, there is a ban on use of calcium carbide incredibly under the PFA Act, 1955, greedy traders silently use this injuriouschemical for several reasons like low cost and ease of availability.

\section{Related Work}

Existing literature uses color features for fruit sorting, recognition, grading, defect identification, maturity detection from the image. One such instance of fruit sorting is explained by $\mathrm{G}$ Feng and $\mathrm{C}$ Qixin[7] in which an smart fruit categorizing structurebased on hue image processingis used. OHTA colour space is used to segment the fruit part from the image and blob abstraction is utilized to discover fruit curve next hueproportion is calculated. HSI color space is used for feature classification which was a major drawback.

A fruit recognition system is proposed in [8] wherepart of the podsection is eliminated from locale section of the image to obtain the right color characteristic values of the fruit. Mean of color from RGB components is calculated and geometrical properties like size, area, perimeter, diameter of the fruit is also taken into consideration and $\mathrm{KNN}$ is applied for classification. Though the method works good computational time taken to calculate geometrical properties served as a major weakness.

$\mathrm{Li}$, Cao, andGuo gave a new method based on foremost color histogram match for fruits categorization in [9]. Here, images of fruitsare acquiredto segment the image.An algorithm to wring pod's image from background in OHTA color space is proposed. The foremostcolor histogram matching is used for hue rating. Experiment has high accuracy up to $97.2 \%$ but it is difficult to meet the needs of real- time application. Anothertechnique called as Direct hueplotting is proposed in [10] for automatic hue ranking of fruit. The suggested approach practices preselected standards to compute a distinctive set of constants for color space transition. Athree-D RGB hue space is transformed into a minor set of color guides which isexceptional to this application acts as a major drawback. Date andtomato maturityapproximation, date surface flaw recognition are estimated by proposed method.

In [11], TRosli,Othman et all have presented computerized fuzzy image analysing system for grading mangoes. The method considerssize, color and skin features of mango for sorting purposes. Then, an algorithm for edge detection is utilized for the study of shapes. Different classes of mango are rated based on fuzzy inference rule. They used high level computational methods for mango grading aided to be a disadvantage. Hong Zheng, Hongfei Lu offeredan algorithm in [12] which is based on Least Square -Support Vector Machine (LS-SVM) in order to discover browning degree of mango using fractal analysis and CIELab parameter. Feature parameters also include $\mathrm{L}^{*} \mathrm{a}^{*} \mathrm{~b}^{*}$ value and fractal dimension (FD) which is obtained from images and median values of the same are considered. Though, LS-SVM classifier built on $\mathrm{L}^{*} \mathrm{a} * \mathrm{~b} *$ and FD delivers $100 \%$ accuracy, consumption of huge data storage space is a major shortcoming.

DameshwariSahu, Ravindra Manohar Potdar presented an innate and stable defect identification and maturity uncovering structure using image processing techniques in 
[13] is projected. Algorithms are built to identify defect and maturity detection of mango fruit, based on solo view fruit images. Mango fruits are tagged into two classes created on the quality ratio[14]. If quality ratio value is bigger than the set ceiling value, the fruit is rotten. On the perverse, if quality ratio value is smaller than the set ceiling value, fruit is nice.Handling bulk data served as a major limitation.

L. Cieplinski in [15] proposed the Dominant Color Descriptor (DCD) for MPEG7 which offersa efficient portrayal of thetypical colours in an image or image section. Its core objective applications arelikenessretrieval in image databases and surfing of image databases based oncolor values which can be several or specific. Contrasting the customary descriptors based on histogram, instead of getting trapped in the color space,the delegate colorsare calculated from every single image, thus letting thecolorillustration to be precise and efficient.

Color is an significantpainterly attributemutuallyfor human vision and computer processing,using DCD plays a vital role in deeper and detailed extraction of colour features for developing a framework to identify natural or artificial ripened mangoes. It provides an efficient extraction andmatching techniquebeyond theextent of the normative elements of the specification for a pragmatic system.

Recently Swati K and Vijay W in [16] proposed a MATLAB based method for detection of artificial ripening of banana fruit. The method uses segmentation to extract colour intensity and texture followed by spatial domain feature extraction to obtain grey level co-occurrence matrix which is used to measure and detect variation in intensity of pixel values. S Maheswaran et all in [17] proposed a device which accepts mango images as input and compares histogram feature values with that of naturally ripened ones and detects artificially ripened mangoes. This method uses smartphones which supports android OS.

\section{Motivation and Contributions}

In the literature though a lot of efforts are focused towards maturity and defect detection of mango fruits focusing on color very few efforts are concentrated towards identification of artificial ripening of mango fruit using color based features. From another perspective deep analysis of mango fruit colors enables us to clearly detect the artificial ripening of mango fruit. To contribute towards this area this paper proposes a computer vision based novel framework using dominant colour features based on MPEG - 7 color descriptors in order toidentifya mango fruit is naturally ripened or artificially ripened. Precisely this paper also suggestsDominant Frequency Image (DFI) constructionand further proposes an algorithm to enhance the accuracy of dominant color extractionfor exact classification of natural or artificial ripened mangoes.

\section{Proposed Framework}

Fig. 1 shows the block diagram of proposed framework which includes three stages namely image preprocessing, dominant colour and feature extraction. In the first stage, the captured images are preprocessed in terms of masking, filtering, segmentation and cropping tasks. In Second stage, for the captured images DFI is generated which clearly indicates dominant colours of given input image as specified in section 4.1 of 
the framework. In the third stage, feature vectors are compared by means of cluster comparison in order to ascertain Natural / Artificial ripeness of fruit images.

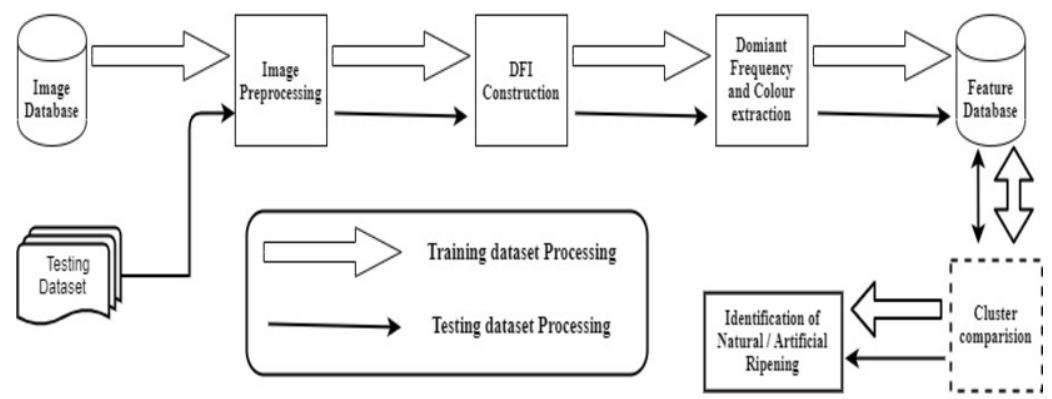

Figure 1. Block diagram of Proposed Framework

\subsection{Construction of Dominant Frequency Image and Dominant colour extraction}

In the proposed framework, first 3D gamut for RGB values of the image is constructed and colour frequencies are computed as given in [18]. In addition to this, Dominant Frequency Image (DFI) representing the frequency of pixels in the original image is generated. Generally DFI construction is needed in order to find dominant colours. In order to improve the accuracy of value extraction from DFI to obtain dominant colours, enhanced algorithm for dominant colour extraction along with DFI construction is also specified as follows for an input image:

The construction of DFI will lead to dominant frequencies of the image with respect to RGB. This dominant frequency will havemaximum frequency values which are mapped on to original image and then exact dominant colours with respect to RGB values are extracted from the enhanced version of the algorithm. This is a iterative process which ends only after primary, secondary and tertiary dominant colours are extracted.

Table 1. Algorithm

Algorithm: DFI construction and Dominant colour extraction

DFI construction.

1. Let Input image $\mathrm{I} € \mathrm{~m} \times \mathrm{n}=0$.

a. For $\mathrm{i}=1$ to $\mathrm{m} ; \mathrm{j}=1$ to $\mathrm{n}$ : do

Freq $=$ gamut $3 \mathrm{D}$

DFI $(m, n)=$ Freq

End For.

b. Calculate Number of colours $=\sum\left(\sum\left(\sum\right.\right.$ (gamut 3D) $\left.)\right)$.

c. Show Number of colours.

d. DFI generated.

Dominant colour extraction

2. Let $\mathrm{Mi}=0,(\mathrm{i} € 1,2,3 \ldots \ldots \mathrm{n})$.

a. For all $\mathrm{Mi}(\mathrm{i} € 1,2,3)$ in DFI

Search I, I $(\mathrm{i}, \mathrm{j})$

If $\mathrm{Mi}=\max (\mathrm{DFI})$

Maxfreq $1=\max (\mathrm{mi})$.

b. DFI (DFI > maxfreqi -1$)$.

c. Map DFI onto I and extract Dominant colour from I 


\subsection{Feature extraction and Cluster comparison}

Distance of a dominant colour is termed as the percentage distribution of a particular dominant frequency which is given by the formula

Distance $\mathrm{n}=$ dominant frequency $\mathrm{n}$ /resolution.

Mean of $\mathrm{R}$ value is the feature considered for further processing as a mango image will have yellow and green colours. In order to know a image is naturally ripened or artificially ripenedfive different clusters where values range from 0 to 255 are considered. The corresponding mapping specifies the ripening evaluation of a given fruit. This is how the prediction of an image is natural or artificial takes place. Results are discussed in the next section.

\section{Results and Discussion}

\subsection{Experimental Setup}

To assess the execution of proposed framework a dataset consisting of mango fruit images is generated for both training and testing purposes. Precisely 60 mangoes belonging to four different types namely Alphonso, Badam, Mallika and Neelam are considered. One set of mangoes are allowed to ripen naturally while the other set of mangoes are artificially ripened using artificial ripening agents like calcium carbide. The mango images are captured by using a Canon power shot digital camera. Fig.2 illustrates few sample snapshots of mangoes with different capturing angles like vertical / horizontal axis, top / side / front views are considered. Around 950 fruit images are generated for both training and testing datasets. Datasets are labelled separately as Naturally Ripened Mango(NRM) fruitsand Artificially Ripened Mango (ARM) fruits. The frame work is developed on a system with following configuration: 4th generation intel i5 processor, $1.7 \mathrm{GHz}$, 64bit Win OS and 8GB RAM, MATLAB version 9.

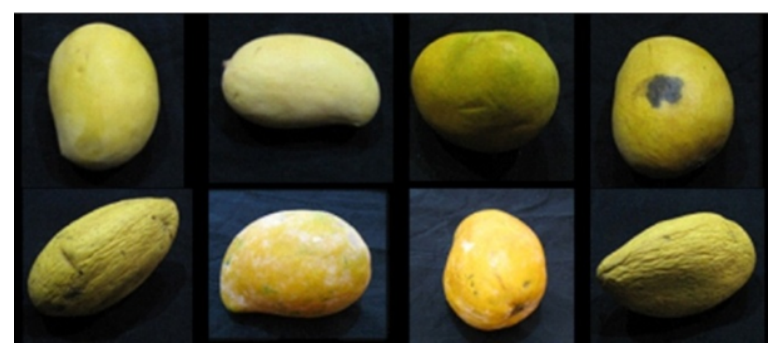

Figure 2. Sample snapshots of mango images (vertical / horizontal axis, top / side / front views.)

\subsection{Experimental Results and discussions}

In order to determine a given sample image is naturally or artificially ripened, dominant colours feature values with respect to RGB is considered which is discussed in section 4.1. Fig.3 shows the graph for colour variations of Primary, Secondary and 
Tertiary Dominant colours of both NRM fruit and ARM fruit images belonging to training dataset. Specifically it is observed that primary dominant colour for naturally ripened fruit varies in the range of 140 - 205 compared to artificial ripening ranging from $210-245$. Secondary and tertiary dominant colours significantly vary with respect to both natural and artificial ripened images.

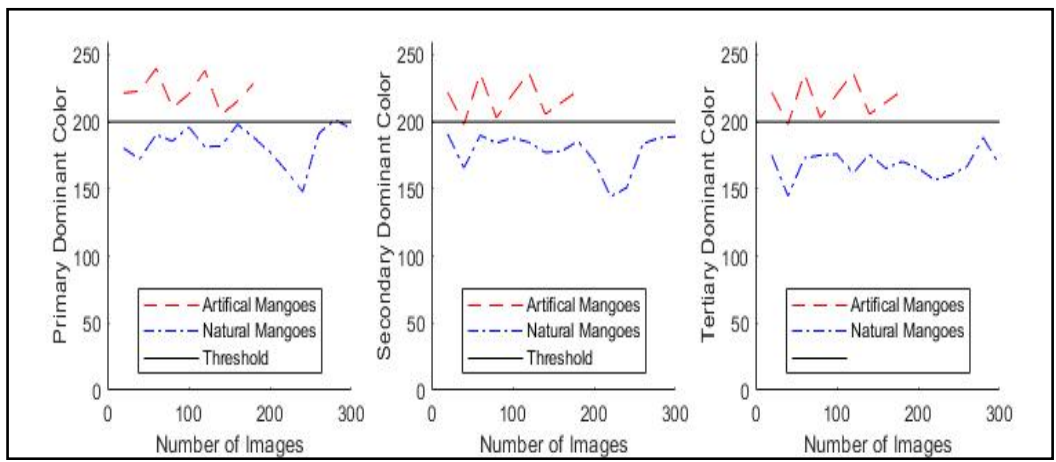

Figure 3. Primary, Secondary and Tertiary Dominant colour variations of training dataset including NRM and ARM images.

Further Table 2 indicates the dominant colour variations of both natural and artificial ripenedfruits in terms of their Min, Mean, Max, Avg and SD. For primary dominant colour mean value of naturally ripened fruit is 183 where as for artificial ripened fruit it is 222. Significant variations in mean values for other two dominant colours is also noticed from the table.

Table 2. Primary, Secondary and Tertiary Dominant colour values of training dataset.

\begin{tabular}{|c|c|c|c|c|c|c|c|c|c|c|c|c|c|c|c|}
\cline { 2 - 13 } \multicolumn{1}{c|}{} & \multicolumn{9}{c|}{ Primary Dominant Colour } & \multicolumn{4}{c|}{ Secondary Dominant Colour } & \multicolumn{4}{c|}{ Tertiary Dominant colour } \\
\cline { 2 - 7 } & Min & Mean & Max & AVG & SD & Min & Mean & Max & AVG & SD & Min & Mean & Max & AVG & SD \\
\hline NRM & 66 & 183 & 248 & 0.24 & 0.06 & 69 & 178 & 240 & 0.17 & 0.03 & 64 & 168 & 224 & 0.13 & 0.02 \\
\hline ARM & 123 & 222 & 255 & 0.18 & 0.04 & 110 & 217 & 255 & 0.15 & 0.03 & 123 & 211 & 255 & 0.11 & 0.01 \\
\hline
\end{tabular}

Fig. 4 shows the graph for colour variations of Primary, Secondary and Tertiary Dominant colours of both NRM and ARM fruit images for testing dataset. Specifically it is observed that primary dominant colour for natural ripeness varies in the range of $150-200$ where as for artificial ripeness it is $205-225$. Secondary and tertiary dominant colours significantly vary with respect to both NRM and ARM images of test dataset. Graphs plotted for both datasets clearly show that natural ripened mangoes values fall below the threshold line where as artificial ripened mangoes values are above the threshold line. 


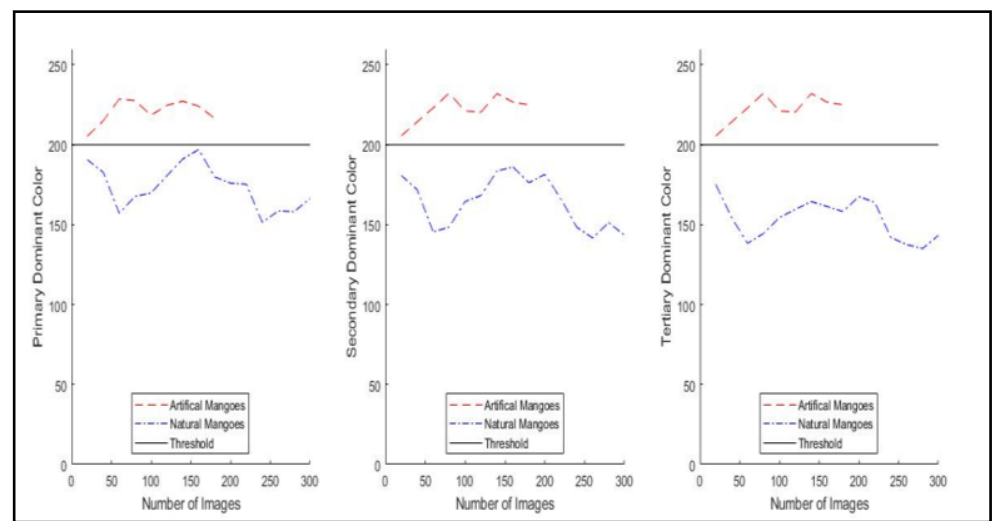

Figure 4. Primary, Secondary and Tertiary Dominant colour variations of testing datasetincluding NRM and ARM images.

Table 3 shows min, mean, max, Avg and SD values for both natural and artificial ripened mango fruits of testing dataset. The primary dominant colour's mean value for natural ripened mango is 154 when compered with artificial ripened it is 214 . A clear difference of mean values for NRM and ARM of testing dataset can be noticed. Both the tables show vital differences in mean, Avg and SD values of primary, secondary and tertiary dominant colours.

Table 3. Primary, Secondary and Tertiary Dominant colour valuesof testing dataset.

\begin{tabular}{|c|c|c|c|c|c|c|c|c|c|c|c|c|c|c|c|}
\hline & \multicolumn{5}{|c|}{ Primary Dominant Colour } & \multicolumn{5}{|c|}{$\begin{array}{l}\text { Secondary Dominant } \\
\text { Colour }\end{array}$} & \multicolumn{5}{|c|}{ Tertiary Dominant colour } \\
\hline & Min & $\begin{array}{l}\mathrm{Me} \\
\text { an }\end{array}$ & $\begin{array}{l}\text { Ma } \\
\mathbf{x}\end{array}$ & AVG & SD & $\begin{array}{l}\text { Mi } \\
\text { n }\end{array}$ & $\begin{array}{l}\mathbf{M} \\
\mathrm{ea} \\
\mathrm{n}\end{array}$ & $\begin{array}{l}\mathrm{M} \\
\mathrm{a} \\
\mathrm{x}\end{array}$ & $\begin{array}{l}\text { AV } \\
\text { G }\end{array}$ & SD & $\begin{array}{l}\text { Mi } \\
\text { n }\end{array}$ & $\begin{array}{l}\text { Mea } \\
\text { n }\end{array}$ & $\begin{array}{l}\mathrm{Ma} \\
\mathrm{x}\end{array}$ & $\begin{array}{l}\text { AV } \\
\text { G }\end{array}$ & SD \\
\hline $\begin{array}{c}\text { NR } \\
\text { M }\end{array}$ & 71 & 154 & 224 & 0.23 & 0.05 & 64 & $\begin{array}{c}16 \\
6\end{array}$ & $\begin{array}{l}2 \\
1 \\
5\end{array}$ & 0.13 & $\begin{array}{c}0.0 \\
2\end{array}$ & 75 & 175 & 215 & 0.12 & 0.02 \\
\hline $\begin{array}{c}\text { AR } \\
\text { M }\end{array}$ & 123 & 214 & 255 & 0.21 & 0.04 & 180 & $\begin{array}{c}22 \\
5\end{array}$ & $\begin{array}{l}2 \\
5 \\
4\end{array}$ & 0.11 & $\begin{array}{c}0.0 \\
1\end{array}$ & $\begin{array}{c}19 \\
3\end{array}$ & 225 & 251 & 0.12 & 0.02 \\
\hline
\end{tabular}

For each of the dominant colour, normalized distance value is calculated by using formula (1). Fig.5 shows graphs for normalized distance values of primary, secondary and tertiary dominant colours obtained from training dataset. Observation is that primary dominant colour has a higher normalized value that ranges from $0.2-0.3$ for natural ripeness where as a lower normalizedvalue ranging from $0.16-0.22$ for artificial ripeness is observed. 


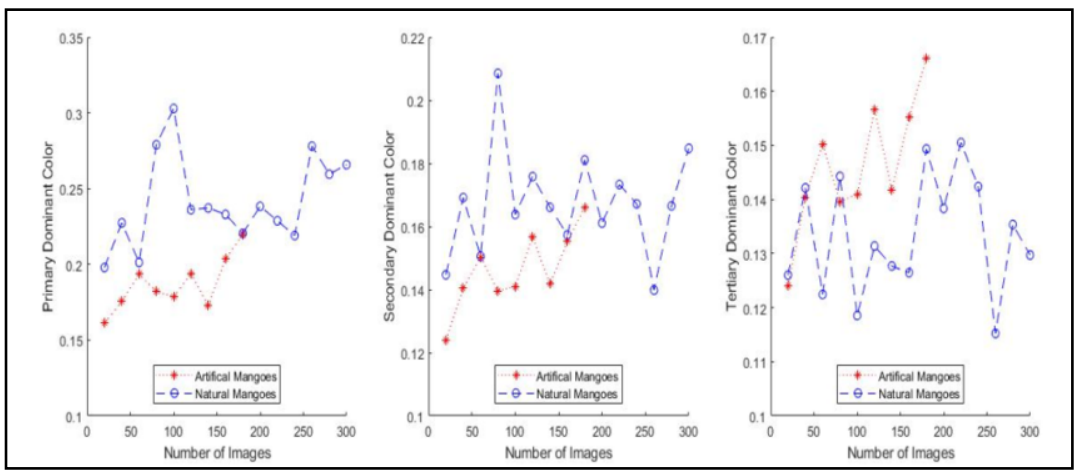

Figure 5. Distance graphs of primary, secondary and tertiary dominant colours for NRM and ARM of training dataset.

Graphs for Normalized distance values of primary, secondary and tertiary dominant colours is shown in Fig.6. Normalized distance values of primary dominant colour for NRM ranges from $0.19-0.27$ where as for ARM it is from $0.17-0.23$.
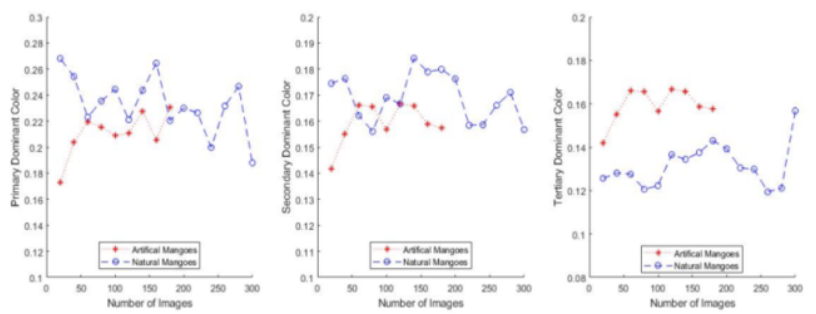

Figure 6. Normalized distance values of primary, secondary and tertiary dominant colours for NRM and ARM images of testing dataset are specified.

Finally, the obtained mean values of dominant colours will be mapped to a cluster to determine whether a sample image is naturally ripened or artificially ripened. Fig.7 shows difference for natural and artificial ripened images of testing dataset. If a sample image maps to cluster 4 then it is NRM image and if mapped to cluster 5 it is ARM image. Note that in testing dataset a negligible percentage of images will cross over the two clusters. Similar kindof cluster mapping is observed in training dataset as well.

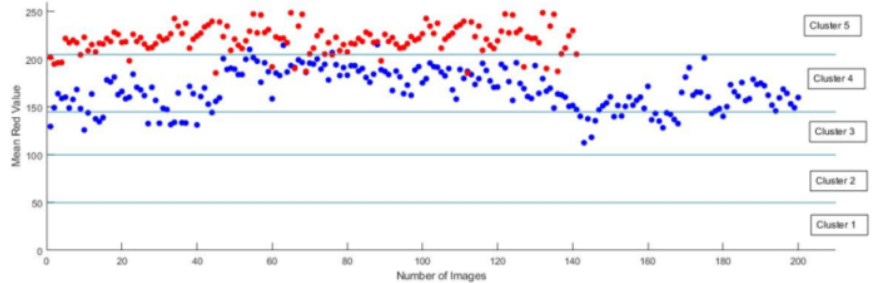

Figure 7. Cluster mapping for NRM and ARM images of testing datasets.

The proposed method gives an admirable results (85.17\% precise). Snapshot of GUI for a given sample image is as shown in fig.8. A User has to select an input image from stored dataset and proceed to obtain the output. Intermediate output images like 
cropped and masked image generated for selected input image are also shown as a part of output. Finally, the classification of sample image to naturally ripened or artificially ripened category is obtained.

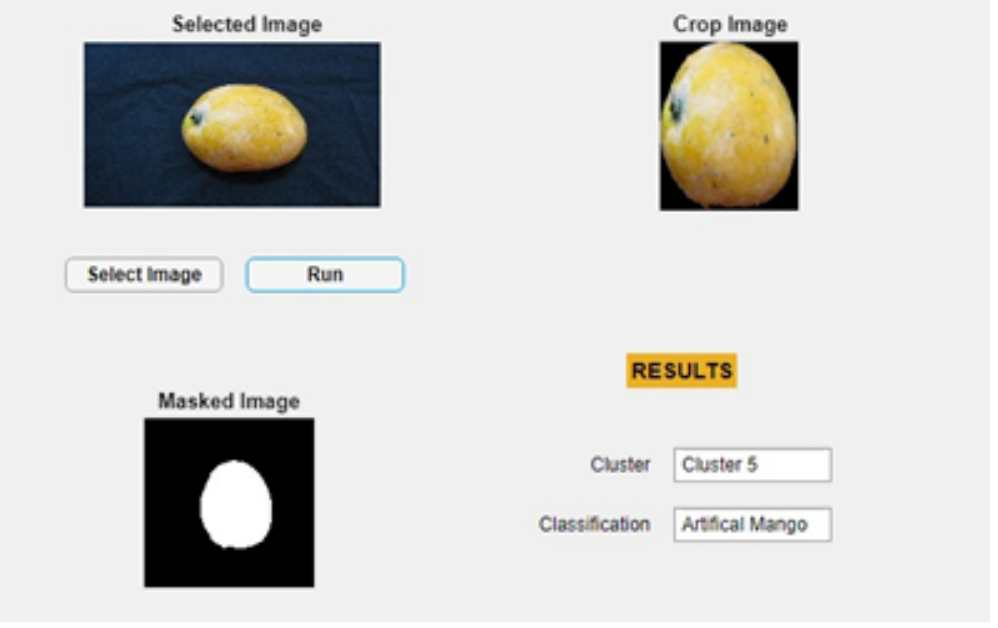

Figure 8. Snapshot of GUI for proposed framework which comprises of input, intermediate results and final output.

\section{6 .Conclusion}

Since fruits are very important part of human diet and mango being the most loved climatic fruit in India it is essential to know if it is naturally ripened or artificially ripened. In this regard the proposed article primarily focuses on dominant colour features described in MPEG-7 to know if a mango is natural or artificial ripened fruit. This can be further extended to other categories of mango fruit.

\section{References}

[1] Ogata J N, Kawani Y, Bevenue A and Casarett L J. 1972. Composition of some mango varieties. Journal of Agriculture Food Chemistry.

[2] Chaudhary T M and Farooqui M A. 1969. Mango processing. Journal of Agriculture Research.

[3] Wills R B H, McGlasson W B, Grahm D and Joyce D C. 2007. Physiology and biochemistry. In Postharvest-An Introduction to the Physiology and Handling of Fruit, Vegetables and Ornamentals, UNSW Press, Sydney, Australia.

[4] Siddiqui M W and Dhua R S. 2010. Eating artificially ripened fruit is harmful. Current Science.

[5] Medlicott A P. 1986. Report on a visit to ITAL Brasil to investigate the effects of maturity, storage and gas treatment on mango fruit ripening. Tropical Dev. and Res. Inst. U.K.

[6] D. Zier, J. -R. Ohm : Common Datasets and Queries in MPEG-7 Color Core Experiments, October 1999. ISO/IEC JTC1/SC29/WG11 MPEG document no. M5060, Melbourne,

[7] Guo Feng and Cao Qixin. Study on Color Image Processing Based Intelligent Fruit Sorting System. Proceedings of the 5thWorld Congress on Intelligent Control and Automation. pp. 4802-4805. June 2004.15-19.

[8] Woo Chaw Seng and SeyedHadiMirisaee. A New Method for Fruits Recognition System. Electrical Engineering and Informatics. August 2009.vol. 01. p. 130-134.

[9] Changyong Li, Qixin Cao, and Feng Guo. A method for color classification of fruits based on machine vision. WTOS 8. February, 2009. vol. 2, p. 312-321. 
[10]Dah-Jye Lee, James K. Archibald, and GuangmingXiong. Rapid Color Grading for Fruit Quality Evaluation Using Direct Color Mapping. IEEE Transactions on Automation Science and Engineering. November 2011.vol. 8, no. 2. pp. 292-302.

[11] TajulRosli,Bin Razak, Mahmod Bin Othman et all. Mango Grading By Using Fuzzy Image Analysis. In proceedings of International Conference on Agricultural, Environment and Biological Sciences, 2012.Phuket.

[12] Hong Zheng and Hongfei Lu. A least-squares support vector machine LS-SVM based on fractal analysis and CIELab parameters for the detection of browning degree on mango. Computers and Electronics in Agriculture. January 2012.vol. 83, p. 47-51.

[13] C. S. Nandi, B. Tudu, and C. Koley. A machine vision-based maturity prediction system for sorting of harvested mangoes. IEEE Trans. Instrum. Meas. vol. 63, no. 7, pp. 1722-1730, 2014.

[14] DameshwariSahu, Ravindra Manohar Potdar. Defect Identification and Maturity Detection of Mango Fruits Using Image Analysis. American Journal of Artificial Intelligence. Vol. 1,.No. 1. 2017. p. 5-14. doi: 10.11648/j.ajai.20170101.12.

[15]L. Cieplinski. Results of Core Experiment CT4 on Dominant Color Extension. ISO/IECJTC1/SC29/WG11 .March 2000 MPEG document no. M5775,Nordwijkerhout .

[16] S.S. Khandarkar, V.R. Wadhankar, D.S. Dabhade.Detection and Identification of Artificially Ripened Fruits Using MATLAB.International Research Journal of Engineering and Technology.2019 Vol.6.No.6,p. $484-487$.

[17] S. Maheswaran, S. Sathesh, P. Priyadharshini, B. Vivek. Identification of artificially ripened fruits using smart phones. 2017.IEEE.

[18] MarkHayworth(2019).ColorFrequencyImage. https://www.mathworks.com/matlabcentral/fileexchange/28164-color-frequency-image.MATLAB Central File Exchange. 